\title{
Relative strength of 5' splice-site strength defines functions of SRSF2 and SRSF6 in alternative splicing of Bcl-x pre-mRNA
}

\author{
Namjeong Choi ${ }^{1}$, Yongchao Liu ${ }^{1}$, Jagyeong $\mathrm{Oh}^{1}$, Jiyeon $\mathrm{Ha}^{1}$, Claudia Ghigna ${ }^{2}$, Xuexiu Zheng ${ }^{1, *} \mathcal{E}^{*}$ Haihong Shen ${ }^{1, *}$ \\ ${ }^{1}$ School of Life Sciences, Gwangju Institute of Science and Technology, Gwangju 61005, Korea, ${ }^{2}$ Institute of Molecular Genetics "Luigi Luca \\ Cavalli-Sforza", National Research Council, Pavia 27100, Italy
}

Bcl-x, a member of the Bcl-2 family, plays a key role in apoptosis. Alternative splicing of Bcl-x pre-mRNA through alternative 5' splice-site selection produces an anti-apoptotic mRNA isoform that includes exon $2 \mathrm{~b}$ and a pro-apoptotic Bcl-x mRNA isoform that excludes exon $2 \mathrm{~b}$. Here we used $\mathrm{Bcl}-\mathrm{x}$ minigene and identified SRSF2 and SRSF6 as two regulatory factors of 5' splice-site selection of Bcl-x pre-mRNA. We selected binding clusters closer to $5^{\prime}$ splice-sites from multiple potential binding sites of SRSF2 and SRSF6 to perform loss of functions analysis through site-directed mutagenesis. Our results demonstrated that these mutations did not abolish regulatory functions of SRSF2 or SRSF6, indicating that a single binding motif or a cluster was not a functional target of these proteins in $\mathrm{BCl}-\mathrm{x}$ pre-mRNA splicing. Random deletion mutagenesis did not disrupt the role of SRSF2 and SRSF6. Importantly, mutagenesis of $5^{\prime}$ splice-site to a conserved or a weaker score demonstrated that the weaker strength of the target $5^{\prime}$ splice-site or higher strength of the other $5^{\prime}$ splice-site strength limited the role of SRSF2 and SRSF6 in $5^{\prime}$ splice-site activation. [BMB Reports 2021; 54(3): 176-181]

\section{INTRODUCTION}

Alternative splicing provides genome diversity. Defects of alternative splicing causes multiple human diseases such as cancer $(1,2)$. Bcl-x, a member of the Bcl-2 family, plays a key role in apoptosis (3). Nearby $5^{\prime}$ splice-site $\left(5^{\prime} \mathrm{L}\right)$ selection of $\mathrm{BCl}-\mathrm{x}$ pre-mRNA produces a Bcl-x longer mRNA isoform that includes exon $2 \mathrm{~b}$, which subsequently directs translation of longer anti-apoptotic $\mathrm{BCl}-\mathrm{x}$ protein isoform (Bcl-xL) (Fig. $1 \mathrm{~A})$

*Corresponding authors. Haihong Shen, Tel: +82-62-715-2507; Fax: +82-62-715-2484; E-mail: haihongshen@gist.ac.kr; Xuexiu Zheng, Tel: +82-62-715-2513; Fax: +82-62-715-2484; E-mail: xuexiuzheng@gist.ac.kr

https://doi.org/10.5483/BMBRep.2021.54.3.170

Received 14 August 2020, Revised 3 September 2020, Accepted 12 October 2020

Keywords: 5' splice-site, Alternative splicing, Bcl-x, SRSF2, SRSF6
(4). By contrast, distant $5^{\prime}$ splice-site (5'SS) activation produces a Bcl-x mRNA isoform that excludes exon $2 b$ which subsequently directs translation of shorter pro-apoptotic Bcl-x protein isoform (BCl-xS) (Fig. 1A). Anti-apoptotic Bcl-xL protein is predominantly expressed in many cancer cells to resist apoptotic stimuli such as chemotherapeutic agents (5). By contrast, pro-apoptotic Bcl-xS causes apoptosis and increase sensitivity to anti-cancer drugs (3). Thus, balanced alternative splicing of Bcl-x plays important roles in the decision of cell survival or cell death. Multiple splicing factors including Sam68, SRSF1, SRSF2, SRSF9 and hnRNP K are shown to regulate alternative splicing of Bcl-x (6).

SRSF2 and SRSF6 are members of arginine/serine-rich (SR) protein family (7). SR proteins share a bipartite structure with two functional domains: an RNA binding domain with multiple RNA recognition motifs (RRMs) and an arginine/serine-rich (RS) domain (7). SR proteins regulate constitutive splicing and alternative splicing. While RNA binding domains provide binding affinity to RNA, RS domain activates splicing (8). Binding motifs of SRSF2 is more degenerate than other SR proteins. Using in vitro functional SELEX, CLIP-seq and structure-based analysis, it was demonstrated that SRSF2 prefers GA- or purinerich and SSNG (S: C/G) $(9,10)$. SRSF6 prefers USCGKM (S: $\mathrm{G} / \mathrm{C}, \mathrm{K}: \mathrm{U} / \mathrm{G}, \mathrm{M}: \mathrm{A} / \mathrm{C}$ ) sequence in in vitro functional SELEX assay (11).

In the present study, using overexpression of various SR proteins with a $\mathrm{Bcl}-\mathrm{x}$ minigene, we identified SRSF2 and SRSF6 as the regulatory factors of $5^{\prime}$ splice-site selection of $\mathrm{BCl}-\mathrm{x}$ pre-mRNA. Among multiple predicted potential binding sites of SRSF2 and SRSF6 in Bcl-x pre-mRNA, we selected binding clusters closer to $5^{\prime}$ splice-sites to perform loss of functions analysis through site-directed mutagenesis. Our results demonstrated that these mutations did not abolish regulatory functions of SRSF2 or SRSF6, indicating that single binding motif or cluster was not a functional target of these proteins in $\mathrm{Bcl}-\mathrm{x}$ pre-mRNA splicing. Random deletion mutagenesis did not disrupt the function of SRSF2 and SRSF6. Importantly, mutagenesis of $5^{\prime}$ splice-site to a conserved or a weaker score demonstrated that weaker strength of target $5^{\prime}$ splice-site or higher strength of the other $5^{\prime}$ splice-site limited the role of SRSF2 and SRS6 in 5' splice-site activation.

ISSN: 1976-670X (electronic edition)

Copyright (C) 2021 by the The Korean Society for Biochemistry and Molecular Biology

c) This is an open-access article distributed under the terms of the Creative Commons Attribution Non-Commercial License (http://creativecommons.org/licenses/by-nc/4.0) which permits unrestricted non-commercial use, distribution, and reproduction in any medium, provided the original work is properly cited. 
A.

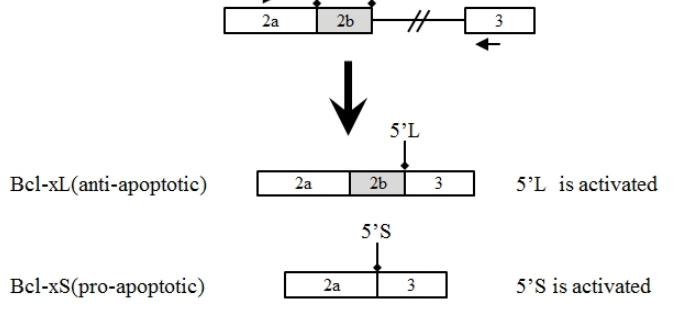

B.

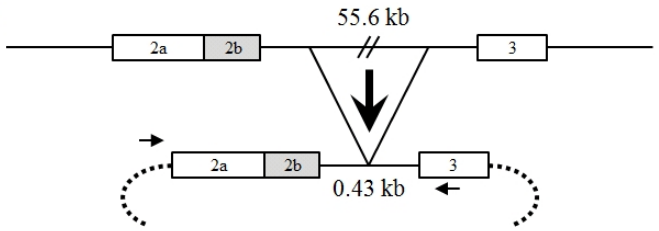

C.

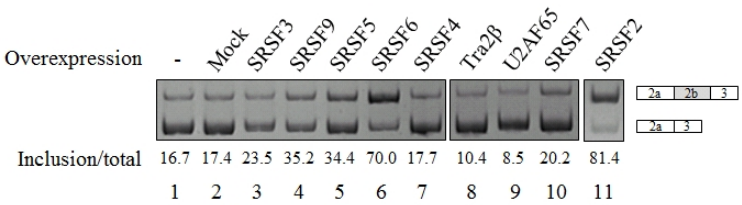

D.

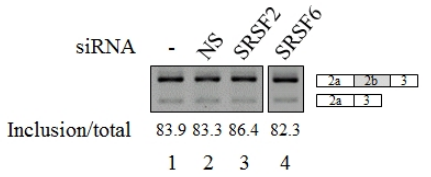

Fig. 1. SRSF2 and SRSF6 promote longer isoform of Bcl-x alternative splicing. (A) Schematic of alternative $5^{\prime}$ splice-site selection in Bcl-x pre-mRNA is shown. Exons are shown with boxes. Introns are shown with lines. Location of $5^{\prime}$ splice-sites of longer $\left(5^{\prime} \mathrm{L}\right)$ and shorter $\left(5^{\prime} \mathrm{S}\right)$ isoforms are shown. Primers used in endogenous RT-PCR are shown by arrows. (B) Schematic of $\mathrm{Bcl}-\mathrm{x}$ minigene is shown. Deleted intron length is shown. Sequences from vector are shown as dotted arc. Primers used in RT-PCR are shown with arrows. (C) RT-PCR assays of $5^{\prime}$ splice-site selection in Bcl-x pre-mRNA within Bcl-x minigene in pcDNA (3.1), SRSF2, SRSF3, SRSF4, SRSF5, SRSF6, SRSF7, SRSF9, $\operatorname{Tra}_{2} \beta$ and $\mathrm{U}^{2} \mathrm{AF}^{65}$ overexpressed cells are shown. Quantitation results are also shown. (D) RT-PCR analysis of $\mathrm{BCl}-\mathrm{x}$ alternative splicing in SRSF2 or SRSF6 knockdown, untreated or non-silencing shRNA treated 293T cells are shown.

\section{RESULTS}

\section{SRSF2 and SRSF6 promote longer isoform of Bcl-x alternative splicing}

To identify the regulatory factors of alternative $5^{\prime}$ splice-site selection in Bcl-x, we applied a minigene-based analysis in 293T cells (Fig. 1A). We produced a Bcl-x minigene in which only 0.43 kilobases $(\mathrm{kb})$ of intron $2(55.6 \mathrm{~kb})$ were included (Fig. 1B). As shown in Fig. 1C (lane 1), the minigene produced much more shorter $\mathrm{Bcl}-\mathrm{x}$ isoform than longer isoform. Thus splicing factors that promote exon inclusion would be easier to be observed with this minigene. In addition, as endogenous $\mathrm{BCl}-\mathrm{x}$ had much more longer isoform than shorter isoform, we expected that splicing factors for promoting longer isoforms in minigene would reduce longer isoform in endogenous $\mathrm{Bcl}-\mathrm{x}$. Considering the fact that there are more longer $\mathrm{Bcl}-\mathrm{x}$ isoforms in cells, detecting reduced longer isoform or increased shorted isoform would be much more apparent. To identify regulatory factors of $\mathrm{BCl}-\mathrm{x} 5^{\prime}$ splice-site selection, we carried out overexpression of various SR proteins along with $\mathrm{BCl}-\mathrm{x}$ minigenes (Fig. 1C). RT-PCR analysis showed that SRSF2 and SRSF6 promoted longer isoform splicing significantly $(\sim 81.4 \%, \sim 70 \%)$ (lanes 11 and 6), whereas SRSF3, SRSF4, SRSF5, SRSF7, SRSF9, $\operatorname{Tra}_{2} \beta$ or $U 2 \mathrm{AF}^{65}$ did not (lanes $3,7,5,10,4,8$ and 9). To assess whether regulatory functions of SRSF2 and SRSF6 in $\mathrm{Bcl}-\mathrm{x}$ minigene were also observable in endogenous $\mathrm{Bcl}-\mathrm{x}$, we performed splicing assays of endogenous $\mathrm{BCl}-\mathrm{x}$ following SRSF2- or SRSF6-targeting shRNA treatment. Fig. 1D shows that reduced expression of SRSF2 and SRSF6 could not alter endogenous $\mathrm{BCl}-\mathrm{x}$ splicing, suggesting an inconsistency of effects between minigene and endogenous alternative splicing. Thus we decided to focus our attention on the regulatory mechanisms of SRSF2 and SRSF6 in Bcl-x minigene.

\section{Multiple potential binding motifs of SRSF2 and SRSF6 are predicted in the $\mathrm{Bcl}-\mathrm{x}$ minigene}

Given the fact that SR proteins would target specific RNA sequences to regulate RNA splicing, we first asked whether there might be potential binding sequences of SRSF2 and SRSF6. Functional SELEX (Systematic Evolution of Ligands by Exponential Enrichment) was applied to identify ESEs (Exon Splicing Enhancers) for SRSF2 and SRSF6 proteins. Sequences of ESEs were described in a web-based program called ESEfinder (10-12) (http://exon.cshl.edu/ESE/). In addition to SELEX based analysis, solution structure analysis and functional confirmation demonstrated that SRSF2 could also recognize SSNG $(\mathrm{S}=\mathrm{C} / \mathrm{G})$ sequences. We then applied $\mathrm{Bcl}-\mathrm{x}$ minigene RNA sequences into ESEfinder tool and SSNG to locate potential binding sequences of SRSF2 and SRSF6. We demonstrated that there are 72 potential SRSF2 (red) and 31 SRSF6 (blue) binding motifs/clusters in Bcl-x pre-mRNA (Supplementary Fig. 1). As the binding motifs of SR proteins especially SRSF2 are degenerate, some of the binding sequences are overlapped. We observed that there are 23 overlapped binding motifs of SRSF2 and SRSF6 (orange) in Bcl-x pre-mRNA. Thus, we conclude that multiple potential binding motifs/clusters exist in Bcl-x pre-mRNA. 


\section{Single binding clusters of SRSF2/SRSF6 are not functional targets of SRSF2/SRSF6 proteins}

Our laboratory and other laboratories have demonstrated that single binding site or clusters are functional targets of SR proteins (13-15). Using previous approaches, we selected sequences located closer to $5^{\prime}$ splice-sites including several binding motifs of "SRSF2" and "SRSF6" in Fig. 2. We performed "loss-of-function" assay for these potential binding sites, in which mutation of potential binding sites of SRSF2 or SRSF6 in $\mathrm{BCl}-\mathrm{x}$ minigene should be able to disrupt the ability of SRSF2 or SRSF6 to promote the longer isoform splicing of Bcl-x. Potential SRSF2 binding sequences we analyzed were located immediately upstream of $5^{\prime}$ splice-site of longer isoform, containing several SRSF2 binding motifs to form a binding cluster (Supplementary Fig. 1 and Fig. 2A, red). We introduced single nucleotide (SRSF2-M1, SRSF2-M2), double nucleotides

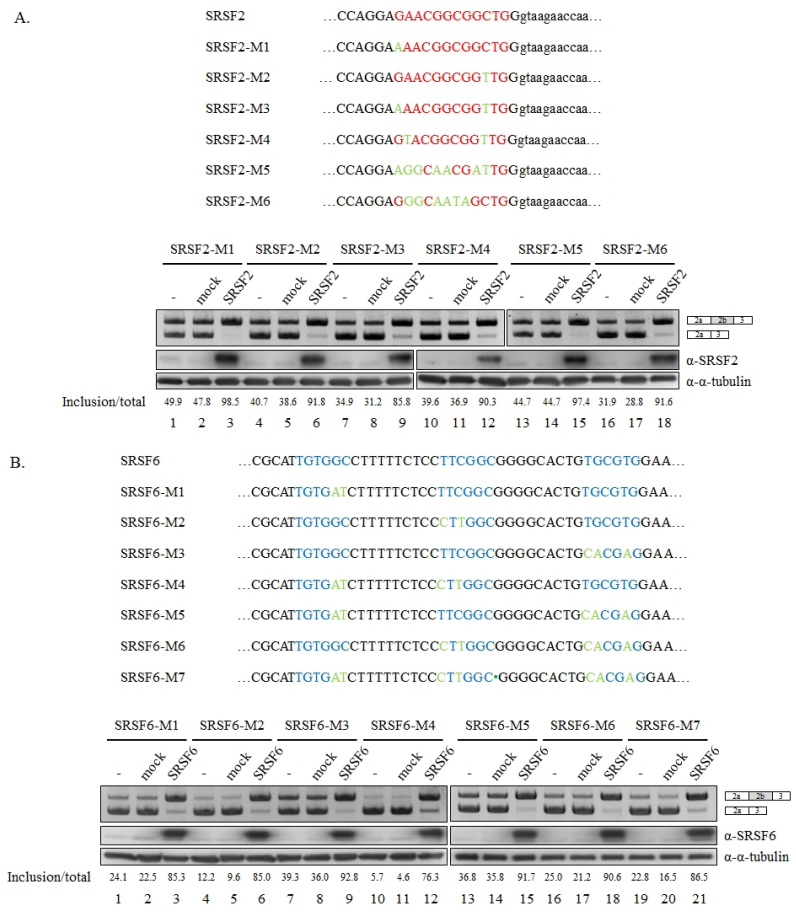

Fig. 2. Single binding clusters of SRSF2/SRSF6 are not functional targets of SRSF2/SRSF6 proteins. (A) (Upper) Mutated sequences in SRSF2-M1, SRSF2-M2, SRSF2-M3, SRSF2-M4, SRSF2-M5 and SRSF2M6 minigenes are shown. Potential SRSF2 binding sequences are shown in red. Mutated sequences are shown in green. (Lower) RT-PCR analysis of $5^{\prime}$ splice-site selection in $\mathrm{Bcl}-\mathrm{x}$ mutant minigenes, including SRSF2-M1, SRSF2-M2, SRSF2-M3, SRSF2-M4, SRSF2-M5, and SRSF2-M6 minigenes are shown. Quantitation results are shown. (B) (Upper) Mutated sequences in SRSF6-M1, SRSF6-M2, SRSF6-M3, SRSF6-M4, SRSF6-M5, SRSF6-M6, and SRSF6-M7 minigenes are shown. Potential SRSF6 binding sequences are shown in blue. Mutated sequences are shown in green. (Lower) RT-PCR analysis of $5^{\prime}$ splice-site selection in Bcl-x mutant minigenes are shown. Quantitation results are shown.
(SRSF2-M3, SRSF2-M4), and multiple nucleotides (SRSF2-M5, SRSF2-M6) into the Bcl-x minigene (green, Fig. 2A). As shown in Fig. 2A, all mutant minigenes still preserved the function of SRSF2 in promoting longer $\mathrm{Bcl}-\mathrm{x}$ isoform, indicating that these mutations did not disrupt the function of SRSF2 (lanes 3, 6, 9, 12, 15 and 18). Thus the RNA sequences did not function as the target of SRSF2. We next carried out mutagenesis analysis of potential SRSF6 binding sites. The sequence we analyzed contained a cluster of three SRSF6 binding sequences sites (blue, Fig. 2B, upper). We produced one binding site (SRSF6-M1, SRSF6-M2, SRSF6-M3), two binding sites (SRSF6-M4, SRSF6-M5, SRSF6-M6) and three binding sites (SRSF6-M7) mutations in $\mathrm{BCl}-\mathrm{x}$ minigene (green, Fig. 3B, upper). As shown in Fig. 2B, none of these mutants disabled the function of SRSF6 in Bcl-x pre-mRNA splicing (lanes 3, 6, 9, 12, 15, 18 and 21). Taken together, these results indicate that binding clusters of SRSF2/SRSF6 did not play as functional targets of SRSF2/SRSF6.

\section{Random deletion of exons did not disrupt functions of SRSF2/SRSF6 in Bcl-x pre-mRNA splicing}

Considering the facts that there are multiple potential binding sequences in $\mathrm{BCl}-\mathrm{x}$ pre-mRNA and that site-directed muta-

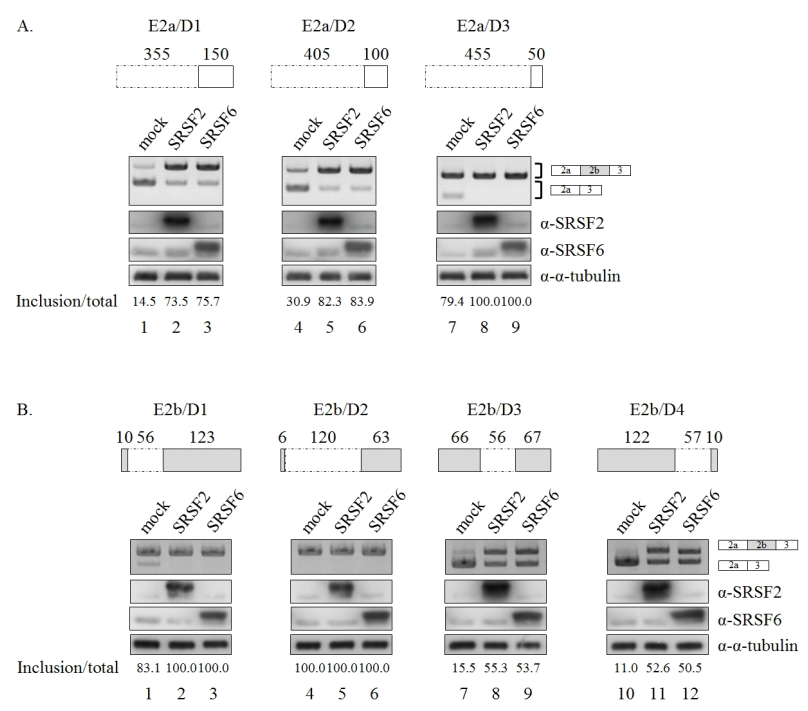

Fig. 3. Random deletion of exons did not disrupt functions of SRSF2 or SRSF6 in Bcl-x pre-mRNA splicing. (A) (Upper) Deleted regions from exon $2 a$ in $E 2 a / D 1, E 2 a / D 2$, and E2a/D3 minigenes are shown with dot lined boxes. Length of the deleted parts are also shown. (Lower) RT-PCR analysis of $5^{\prime}$ splice-site selection in E2a/D1, E2a/D2 and E2a/D3 minigenes are shown. Quantitation results are shown. (B) (Upper) Deleted regions from exon $2 \mathrm{~b}$ in $\mathrm{E} 2 \mathrm{~b} / \mathrm{D} 1, \mathrm{E} 2 \mathrm{~b} / \mathrm{D} 2, \mathrm{E} 2 \mathrm{~b} / \mathrm{D} 3$ and E2b/D4 minigenes are shown with dot lined boxes. Length of the deleted parts are also shown. (Lower) RT-PCR analysis of $5^{\prime}$ splice-site selection in E2b/D1, $E 2 b / D 2, E 2 b / D 3$ and E2b/D4 minigenes are shown. Quantitation results are shown. 
genesis of SRSF2/SRSF6 binding clusters could not identify functional targets of these proteins, we decided to test the possibility that SRSF2 and SRSF6 might function through more binding motifs or clusters. We first deleted $355 \mathrm{nt}, 405 \mathrm{nt}$ and $455 \mathrm{nt}$ from exon 2a (E2a/D1, E2a/D2 and E2a/D3) (Fig. 3A, Upper). As shown in Fig. 3A, SRSF2 and SRSF6 were still able to promote longer form expression of E2a/D1 and E2a/D2 mutants (lanes 2, 3, 5 and 6, lower). E2a/D3 mutant produced E2b included isoform exclusively (lane 7), an increase of the inclusion isoform could not be observed (lanes 8 and 9). Thus, E2a deletion mutation was unable to identify the functional target of SRSF2 or SRSF6. We further performed deletion mutagenesis of exon $2 \mathrm{~b}$ by deleting $56 \mathrm{nt}, 120 \mathrm{nt}$ at $5^{\prime}$ of exon $2 b(E 2 b / D 1$ and E2b/D2), 56 nt from middle (E2b/D3), and 57 nt from $3^{\prime}$ of exon $2 b$ (E2b/D4) (Fig. 3B, Upper). Among these mutants, E2b/D2 mutant produced E2b-included isoform exclusively (lane 4, Fig. 3B, lower), making it difficult to detect the abolishment of functions of SRSF2 and SRSF6 (lanes 5 and 6). Three other mutants showed strong activity of SRSF2 and SRSF6 on exon 2b inclusion (lanes 2, 3, 8, 9, 11 and 12). Thus, random deletions of exon $2 \mathrm{~b}$ were unable to identify functional targets of SRSF2/SRSF6. These results imply that a combination of binding sites from different locations is needed to be functionally targeted by SRSF2 and SRSF6.

\section{Relative strength of $5^{\prime}$ splice-site strength determines splices-site selection functions of SRSF2 and SRSF6}

We observed that $5^{\prime}$ splice-site of E2b (5'2b) (90.83) had a much higher strength than E2a (5'2a) (77.41) (Human Splicing Finder, http://umd.be/Redirect.html) (16). We thus wondered whether $5^{\prime}$ splice-site strength might play important roles in the $5^{\prime}$ splice-site selection function of SRSF2 and SRSF6. We first produced a mutant minigene in which $5^{\prime} 2 \mathrm{a}$ and $5^{\prime} 2 \mathrm{~b}$ were inverted (Inv) (Fig. 4A, left). Strikingly, activities of SRSF2 and SRSF6 in promoting exon $2 \mathrm{~b}$ inclusion were almost abolished (lanes 2 and 3, right). Thus, 5' splice-site strength plays a role in functions of SRSF2 and SRSF6. We next performed mutagenesis for the $5^{\prime}$ splice-site to assess effects of the strength of $5^{\prime}$ splice-site on splice-site selection. We first mutated 5'2a sequences without changing $5^{\prime} 2 \mathrm{~b}$ sequences. We produced a conservative or a weaker score (71.60) mutations in 5'2a (Cons2a and W2a) (Fig. 4B, left). As shown in Fig. 4B, Cons2a almost completely abolished activities of SRSF2 and SRSF6 (lanes 2 and 3), indicating that $5^{\prime}$ splice-site with higher score could overcome activation of the other $5^{\prime}$ splice-site by SRSF2 and SRSF6. W2a mutant was spliced to form the longer Bcl-x isoform exclusively. Thus increase of longer isoform by SRSF2 and SRSF6 could not be observed. We further mutated $5^{\prime} 2 \mathrm{~b}$ sequences without mutating 5'2a sequences. SRSF2 and SRSF6 promoted E2b inclusion of Cons $2 \mathrm{~b}$ mutant (Fig. 4C, right, lanes 2 and 3), in which $5^{\prime} 2 \mathrm{~b}$ was mutated to a conserved sequence (Fig. 4C, left), suggesting that better splice-site did not interrupt its activation by SRSF2 or SRSF6. By contrast, W2b mutant abolished SRSF2 and SRSF6 (lanes 5 and 6),

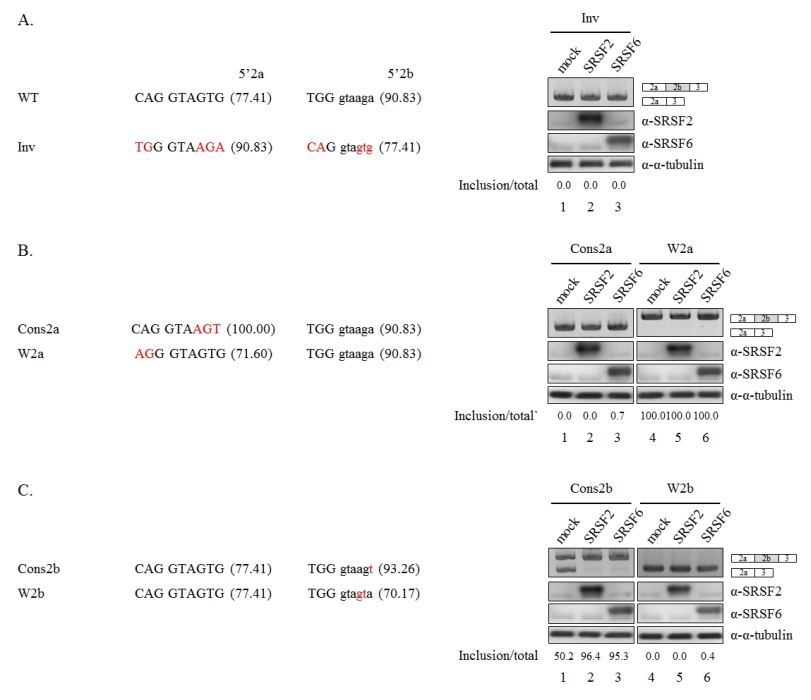

Fig. 4. Relative $5^{\prime}$ splice-site strength determines splices-site selection functions of SRSF2 and SRSF6. (A) (Upper) Two $5^{\prime}$ sequences of $5^{\prime}$ splice-site switched mutant minigene (Inv) are shown. Mutated sequences are shown in red. (Lower) RT-PCR analysis results of $5^{\prime}$ splice-site selection in Inv minigenes are shown. (B) (Upper) Two 5' sequences of Cons2a and W2a minigenes are shown. Mutated sequences are shown in red. (Lower) RT-PCR analysis results of $5^{\prime}$ splice-site selection in Cons2a and W2a minigenes are shown. (C) (Upper) Two 5' sequences of Cons2b and W2b minigenes are shown. Mutated sequences are shown in red. (Lower) RT-PCR analysis of $5^{\prime}$ splice-site selection in Cons2b and W2b minigenes are shown.

indicating that weaker $5^{\prime}$ splice-site was not able to support its activation by SRSF2 and SRSF6. Taken together, we conclude that, for $5^{\prime}$ splice-site selection activity of SRSF2 and SRSF6, mutagenesis of $5^{\prime}$ splice-site to a conserved or a weaker score demonstrated that an activated $5^{\prime}$ splice-site with weaker score or the other $5^{\prime}$ splice-site with a higher score could limit the role of $5^{\prime}$ splice-site.

\section{DISCUSSION}

In this study, we identified SRSF2 and SRSF6, as regulatory proteins for $5^{\prime}$ splice-site selection of Bcl-x pre-mRNA using a minigene system. We applied site-directed mutagenesis to potential binding motifs or clusters that located closer to the $5^{\prime}$ splice-site to identify target RNA sequences of SRSF2 and SRSF6. We further performed random deletion mutagenesis of exons $2 a$ and $2 b$ to locate targets of these proteins. Our results indicate that both site-directed and deletion mutations could not abolish activities of Bcl-x. Remarkably, the strength of 5' splice-site of the one activated by SRSF2 and SRSF6 and the other one play roles in functions of SRSF2 and SRSF6. Less conserved sequences of activated $5^{\prime}$ splice-site or more conserved sequences of the other one abolished activities of SRSF2 and SRSF6 in Bcl-x splicing, suggesting that the strength 
of $5^{\prime}$ splice-site could regulates the function of SRSF2 and SRSF6.

SR proteins have a single RNA binding motif or cluster in splicing regulation $(15,17)$. We observed that mutations of single binding motif or cluster in Bcl-x pre-mRNA were unable to disrupt functions of SRSF2 or SRSF6. Deletion mutations of exon $2 \mathrm{a}$ and $2 \mathrm{~b}$ were unable to abolish their functions either, suggesting that multiple binding motifs or clusters from different locations might function as functional targets of SRSF2 and SRSF6. It has been shown that SR proteins collaborate in the regulation of alternative splicing $(18,19)$. Splicing machineries in multiple binding motifs could collaborate to regulate $\mathrm{Bcl}-\mathrm{x}$ splicing. In $\mathrm{Bcl}-\mathrm{x}$ pre-mRNA, many binding motifs or clusters of SRSF2 and SRSF6 were predicted. From these motifs or clusters, we selected sequences closer to the $5^{\prime}$ splice-site to perform functional analysis. These mutations were not functional targets of SRSF2 or SRSF6. Our previous results have demonstrated that single binding targets of SR proteins are located close to splice-sites $(13,17)$. Although we could not test all predicted binding motifs for functional analysis, distant sequences from splice-sites might also be functional targets of SRSF2 and SRSF6.

Although SRSF2 and SRSF6 regulate Bcl-x alternative splicing in a minigene assay with an overexpression approach, these proteins could not modulate endogeneous $\mathrm{Bcl}-\mathrm{x}$ premRNA using shRNA-mediated knockdown approach. Differences in the overexpression of exogenous SR proteins and endogenous knockdown can explain the disparity in results using different approaches. Such disparity might also come from differences of $\mathrm{Bcl}-\mathrm{x}$ RNA. The $\mathrm{Bcl}-\mathrm{x}$ minigene we tested did not include the whole intron $(55.6 \mathrm{~kb})$, but only partial intron $(0.43 \mathrm{~kb})$. It has been shown that overexpressed SRSF2 can regulate endogenous $\mathrm{Bcl}-\mathrm{x}$ pre-mRNA splicing in A549 cells (20). However, we could not observe effects of SRSF2 effects on endogenous Bcl-x in the present study. Such differences between the previous study and the present study could be due to experimental differences and different cell lines tested.

Our results demonstrated the importance of $5^{\prime}$ splice-site strength in the function of SRSF2 and SRSF6 in regulating Bcl-x pre-mRNA splicing. This is consistent with previous reports showing that alternative $5^{\prime}$ splice-site is affected by the strength of $5^{\prime}$ splice-site and the distance between two $5^{\prime}$ splice-sites (21-23). $5^{\prime}$ splice-site selection has various selection mechanisms. One study has shown that distant $5^{\prime}$ splicesite affects proximal $5^{\prime}$ splice-site, while another report has shown that spliceosome prefers most proximal $5^{\prime}$ splice-site to distal $5^{\prime}$ splice-site $(24,25)$. Although strength of both distant and proximal $5^{\prime}$ splice-sites play important roles in the function of SRSF2 and SRSF6, they have opposite directions. How the strength of $5^{\prime}$ splice-site affects functions of SRSF2 and SRSF6 need to be clarified through further studies.

\section{MATERIALS AND METHODS}

\section{Plasmid construction}

$\mathrm{Bcl}-\mathrm{x}$ minigene was produced by deleting part of exon 2a and intron from the minigene that we constructed previously (26). Nhe I and EcoR I restriction enzymes were used to clone Bcl-x minigene into pCl-neo vector. All mutant constructs were generated by site-directed mutagenesis using $\mathrm{Bcl}-\mathrm{x}$ minigene as a template. All primer sequences are listed in Supplementary Table 1.

\section{Cell culture, plasmid transfection and immunoblotting}

HEK293T cells were grown in Dulbecco's Modified Eagle's Medium (DMEM) media (HyClone) supplemented with $10 \%$ Fetal Bovine Serum (FBS) in a $5 \% \mathrm{CO}_{2}$ incubator at $37^{\circ} \mathrm{C}$. $2 \mathrm{mM}$ Glutamine, $100 \mathrm{U} / \mathrm{ml}$ penicillin and $100 \mu \mathrm{g} / \mathrm{ml}$ streptomycin were also added. To transfect DNA into cells, cells are treated with $100 \mu \mathrm{l}$ DMEM containing $0.5 \mu \mathrm{g}$ DNA mixed with $2 \mu \mathrm{g}$ polyethyleneimide (PEI). Culture media were changed after $4 \mathrm{~h}$ of incubation. RNAs were extracted from cells at $48 \mathrm{~h}$ after transfection. Immunoblotting was performed as previously described (27) using anti-SC35 (Millipore), anti-SRp55 (Millipore) and anti-Tubulin (Abcam) antibodies.

\section{RT-PCR}

RT-PCR was performed as previously described (28). MLV reverse transcriptase (ELPISBIO) and oligo-dT18 primer were used to reverse transcribe RNA. In the PCR reaction, primer set $\mathrm{bm} 1 / \mathrm{bm} 2$ was used analyze $\mathrm{Bcl}-\mathrm{x}$ minigene splicing while primer set be1/be2 was used to detect endogenous $\mathrm{BCl}-\mathrm{x}$ splicing.

\section{AUTHOR CONTRIBUTIONS}

H.S., X.Z. and C.G. designed the concept of the present study and supervised it. N.C., Y.L., J.O., J.H. acquired, analyzed and interpreted the data. X.Z. and H.S. wrote the manuscript. All authors read and approved the manuscript.

\section{ACKNOWLEDGEMENTS}

This work was supported by the following grants: NRF2020R1A2C2004682 grants to Haihong Shen, NRF-2019R1I 1A1A01057372 grant to Xuexiu Zheng, and Cell Logistics Research Center (grant No. 2016R1A5A1007318) funded by the Ministry of Education and National Research Foundation of Korea. This work was also supported by "GIST Research Institute (GRI) ARI" grant funded by the GIST in 2020.

\section{CONFLICTS OF INTEREST}

The authors have no conflicting interests. 


\section{REFERENCES}

1. Wang L, Brooks AN, Fan J et al (2016) Transcriptomic Characterization of SF3B1 Mutation Reveals Its Pleiotropic Effects in Chronic Lymphocytic Leukemia. Cancer Cell 30, 750-763

2. Yin S, Gambe RG, Sun J et al (2019) A Murine Model of Chronic Lymphocytic Leukemia Based on B Cell-Restricted Expression of Sf3b1 Mutation and Atm Deletion. Cancer Cell 35, 283-296 e285

3. Sumantran VN, Ealovega MW, Nunez G, Clarke MF and Wicha MS (1995) Overexpression of Bcl-XS sensitizes MCF-7 cells to chemotherapy-induced apoptosis. Cancer Res 55, 2507-2510

4. Mercatante DR, Bortner CD, Cidlowski JA and Kole R (2001) Modification of alternative splicing of BCl-x pre-mRNA in prostate and breast cancer cells. analysis of apoptosis and cell death. J Biol Chem 276, 16411-16417

5. Espana L, Fernandez Y, Rubio N, Torregrosa A, Blanco J and Sierra A (2004) Overexpression of Bcl-xL in human breast cancer cells enhances organ-selective lymph node metastasis. Breast Cancer Res Treat 87, 33-44

6. Garneau D, Revil T, Fisette JF and Chabot B (2005) Heterogeneous nuclear ribonucleoprotein $\mathrm{F} / \mathrm{H}$ proteins modulate the alternative splicing of the apoptotic mediator Bcl-x. J Biol Chem 280, 22641-22650

7. Graveley BR (2000) Sorting out the complexity of SR protein functions. RNA 6, 1197-1211

8. Shen H and Green MR (2006) RS domains contact splicing signals and promote splicing by a common mechanism in yeast through humans. Genes Dev 20, 1755-1765

9. Daubner GM, Clery A, Jayne S, Stevenin J and Allain FH (2012) A syn-anti conformational difference allows SRSF2 to recognize guanines and cytosines equally well. EMBO J 31, 162-174

10. Cartegni L, Wang J, Zhu Z, Zhang MQ and Krainer AR (2003) ESEfinder: A web resource to identify exonic splicing enhancers. Nucleic Acids Res 31, 3568-3571

11. Liu HX, Zhang M and Krainer AR (1998) Identification of functional exonic splicing enhancer motifs recognized by individual SR proteins. Genes Dev 12, 1998-2012

12. Liu HX, Chew SL, Cartegni $L$, Zhang $M Q$ and Krainer $A R$ (2000) Exonic splicing enhancer motif recognized by human SC35 under splicing conditions. Mol Cell Biol 20, 1063-1071

13. Moon H, Cho S, Loh TJ et al (2014) SRSF2 promotes splicing and transcription of exon 11 included isoform in Ron proto-oncogene. Biochim Biophys Acta 1839, 11321140

14. Jang HN, Lee M, Loh TJ et al (2014) Exon 9 skipping of apoptotic caspase-2 pre-mRNA is promoted by SRSF3 through interaction with exon 8. Biochim Biophys Acta
1839, 25-32

15. Cartegni L and Krainer AR (2002) Disruption of an SF2/ASF-dependent exonic splicing enhancer in SMN2 causes spinal muscular atrophy in the absence of SMN1. Nat Genet 30, 377-384

16. Desmet FO, Hamroun D, Lalande M, Collod-Beroud G, Claustres M and Beroud C (2009) Human Splicing Finder: an online bioinformatics tool to predict splicing signals. Nucleic Acids Res 37, e67

17. Jang HN, Liu Y, Choi N et al (2018) Binding of SRSF4 to a novel enhancer modulates splicing of exon 6 of Fas pre-mRNA. Biochem Biophys Res Commun 506, 703-708

18. Crawford JB and Patton JG (2006) Activation of alphatropomyosin exon 2 is regulated by the SR protein 9G8 and heterogeneous nuclear ribonucleoproteins $\mathrm{H}$ and $\mathrm{F}$. Mol Cell Biol 26, 8791-8802

19. Lynch KW and Maniatis T (1996) Assembly of specific SR protein complexes on distinct regulatory elements of the Drosophila doublesex splicing enhancer. Genes Dev 10, 2089-2101

20. Merdzhanova G, Edmond V, De Seranno S et al (2008) E2F1 controls alternative splicing pattern of genes involved in apoptosis through upregulation of the splicing factor SC35. Cell Death Differ 15, 1815-1823

21. Yu Y, Maroney PA, Denker JA et al (2008) Dynamic regulation of alternative splicing by silencers that modulate 5' splice site competition. Cell 135, 1224-1236

22. Fisette JF, Toutant J, Dugre-Brisson S, Desgroseillers L and Chabot B (2010) hnRNP A1 and hnRNP H can collaborate to modulate 5' splice site selection. RNA 16, 228-238

23. Bielli P, Bordi M, Di Biasio V and Sette C (2014) Regulation of BCL-X splicing reveals a role for the polypyrimidine tract binding protein (PTBP1/hnRNP I) in alternative 5 ' splice site selection. Nucleic Acids Res 42, 12070-12081

24. Reed R and Maniatis T (1986) A role for exon sequences and splice-site proximity in splice-site selection. Cell 46, 681-690

25. Hicks MJ, Mueller WF, Shepard PJ and Hertel KJ (2010) Competing upstream $5^{\prime}$ splice sites enhance the rate of proximal splicing. Mol Cell Biol 30, 1878-1886

26. Lee J, Zhou J, Zheng $X$ et al (2012) Identification of a novel cis-element that regulates alternative splicing of Bcl-x pre-mRNA. Biochem Biophys Res Commun 420, 467-472

27. Cho S, Moon H, Loh TJ et al (2015) Splicing inhibition of U2AF65 leads to alternative exon skipping. Proc Natl Acad Sci U S A 112, 9926-9931

28. Moon H, Jang HN, Liu $\mathrm{Y}$ et al (2019) RPM but not the Asp/Glu domain of hnRNP C1/C2 is required for splicing regulation of Ron exon 11 pre-mRNA. BMB Rep 52, 641-646 